\title{
Les comportements langagiers dans les groupes de jeunes en milieu urbain
}

Le cas de la ville de Ouagadougou

Language Behaviours of Youth Group in Urban Area. The Case of Ouagadougou

\section{Abou Napon}

\section{CpenEdition}

\section{Journals}

Édition électronique

URL : http://journals.openedition.org/etudesafricaines/116

DOI : 10.4000/etudesafricaines. 116

ISSN : 1777-5353

Éditeur

Éditions de l'EHESS

Édition imprimée

Date de publication : 1 janvier 2001

Pagination : 697-710

ISBN : 978-2-7132-1394-6

ISSN : 0008-0055

\section{Référence électronique}

Abou Napon, « Les comportements langagiers dans les groupes de jeunes en milieu urbain », Cahiers d'études africaines [En ligne], 163-164 | 2001, mis en ligne le 21 novembre 2013, consulté le 05 février 2021. URL : http://journals.openedition.org/etudesafricaines/116 ; DOI : https://doi.org/10.4000/ etudesafricaines. 116 


\title{
Abou Napon
}

\section{Les comportements langagiers dans les groupes de jeunes en milieu urbain}

\author{
Le cas de la ville de Ouagadougou
}

Toutes les institutions administratives, politiques et économiques du Burkina Faso sont rassemblées à Ouagadougou, fief de l'ethnie moaga (près de $48 \%$ de la population du pays). La configuration ethnolinguistique de cette ville a changé avec l'arrivée des migrants à la recherche d'emplois rémunérés et, de ce fait, Ouagadougou est devenu une ville multilingue où l'on rencontre des représentants des différents groupes ethniques qui composent la nation burkinabè.

Le français (langue officielle du pays), le moore (langue des autochtones de la cité), le dioula (langue véhiculaire interethnique) et les langues grégaires (utilisées principalement en milieu familial) sont les principales langues employées pour la communication.

Dans cette situation de contact de langues - français/langues nationales et langues nationales entre elles - , notre préoccupation est, d'une part, de voir comment se fait la gestion des langues en milieu urbain et, d'autre part, de cerner les représentations que les jeunes, tiraillés entre la tradition et la modernité, ont des langues à Ouagadougou. Leurs comportements langagiers permettent de nous éclairer sur la manière dont se fait la gestion des langues nationales et du français dans les villes modernes.

Les hypothèses que nous avançons sont les suivantes: les usages au sein des groupes des jeunes sont les mêmes d'un secteur ${ }^{1}$ à un autre; les représentations que les jeunes ont des langues sont tributaires des rapports de force qui existent entre le français et les langues nationales.

Notre étude, qui porte sur le contact des langues, s'inscrit dans les domaines de la sociolinguistique, du bilinguisme et de la diglossie. Nos investigations ont porté sur les groupes de jeunes qui se retrouvent tous les soirs pour boire du thé. Ces groupes sont appelés « les grains » en référence

1. Le secteur est une entité administrative composée d'au moins une dizaines de quartiers. La ville de Ouagadougou est subdivisée en trente secteurs. 
aux grains de thé. Cette appellation, aux dires des jeunes, aurait pour origine la ville de Bobo-Dioulasso, capitale économique du pays, où ces jeunes ont l'habitude de prendre le thé tous les jours. C'est ensuite que le terme est arrivé à Ouagadougou notamment par l'intermédiaire des élèves.

Nous n'avons retenu que trois groupes de jeunes, mais notre observation a porté au départ sur six groupes dont les choix ont été faits selon les critères suivants :

- la composition ethnique composite ;

- la composition socioculturelle (scolarisés/non scolarisés);

- la connaissance d'au moins un membre du groupe ;

- le nombre de membres du groupe (au moins vingt personnes).

Le premier critère avait pour objet de mesurer l'influence de l'ethnie sur les pratiques langagières au sein des groupes. Nous avons pris en compte également le niveau de scolarisation dans le choix des groupes pour appréhender l'impact de la scolarisation dans le choix des langues. Le fait de connaître un membre du groupe a été déterminant, car il a permis au groupe de se sentir en sécurité. En effet, il existe à Ouagadougou, à propos des enquêtes, une psychose due au climat sociopolitique national tendu. Aussi, toute enquête, quelle que soit sa nature, est perçue comme un interrogatoire policier. Beaucoup de gens se méfient ainsi des enquêteurs. Nous nous sommes intéressés aux groupes constitués d'au moins vingt personnes pour être sûr d'avoir des pratiques langagières variées, et ce, compte tenu du nombre des interactants.

Notre enquête de terrain s'est déroulée en trois phases. La première nous a permis de choisir les groupes sur lesquels devait porter l'observation. Comme nous l'avons souligné plus haut, six groupes ont été visités dans les secteurs $12,6,9,14,15,16$. Mais en définitive, nous n'avons retenu que trois groupes situés dans les trois derniers secteurs $(14,15$ et 16), car c'étaient les seuls qui répondaient aux critères qui avaient été préalablement établis.

La technique utilisée, la méthode participante, consistait à se rendre auprès des groupes de jeunes et à passer une soirée avec eux, afin de cerner la composition et le fonctionnement des groupes. Une fois les trois groupes retenus, la deuxième phase de l'enquête permettait d'enregistrer de manière directe les pratiques langagières au sein des groupes à l'aide d'un magnétophone dissimulé sous nos vêtements. L'objectif était de recueillir les comportements langagiers des jeunes dans un cadre naturel. Nous avons pensé que le fait de se savoir enregistré aurait pu amener les locuteurs à modifier leurs habitudes langagières.

Après cette étape qui devait nous permettre d'observer les usages des langues au sein des groupes, nous avons interrogé six jeunes (soit deux par groupe) pour recueillir des informations sur les points suivants :

- la structuration et le fonctionnement des groupes;

- les raisons qui déterminent le choix des langues au sein des groupes;

- les représentations des langues. 
Pour la transcription de notre corpus, nous avons adopté les conventions suivantes : $\mathrm{L}=$ locuteur $; \mathrm{E}=$ enquêté $; \mathrm{LM}=$ locuteur du moore $; \mathrm{LD}=$ locuteur du dioula.

\section{Structuration et fonctionnement des groupes}

Le groupe du secteur 14 (groupe 1) est composé de jeunes appartenant à des groupes ethniques différents (mossi, gourounsi, dioula, peul). Leur âge se situe entre vingt et trente ans et leurs niveaux d'instruction sont variés : non scolarisés, peu scolarisés et personnes dont le niveau d'instruction se situe entre la sixième de l'école secondaire et la maîtrise (université). Ils se retrouvent tous les soirs, au domicile d'un de leurs camarades, pour boire du thé et échanger des propos sur les problèmes quotidiens de la vie afin de trouver ensemble des solutions. Les thèmes abordés sont, entre autres, le chômage des jeunes, la politique, les problèmes de sexe, etc. Tous les meubles de la maison (bancs, chaises, tables) sont mis à leur disposition pour leur permettre de se retrouver dans cette famille. Ainsi, chaque membre du groupe se comporte dans cette cour comme s'il était chez lui. L'esprit cultivé au sein de ce groupe est donc celui de la famille au sens large du terme. Ce groupe se présente également comme un refuge pour tous les membres désirant se détendre. On y vient pour rire ou pour recevoir les conseils des autres. Sa création remonte à l'adolescence. Les membres se fréquentent depuis leur jeune âge (c'est-à-dire depuis l'école primaire). Ils se connaissent presque tous et sont imprégnés des problèmes de chacun d'entre eux.

Le groupe du secteur 15 (groupe 2) comprend des personnes dont l'âge varie entre 20 et 40 ans. On y retrouve à quelques exceptions près les mêmes ethnies que celles citées dans le groupe du secteur 14: mossi, gourounsi, bissa, samo, peul, dagara, dioula. Contrairement au premier groupe constitué d'élèves, d'étudiants et de quelques travailleurs, le deuxième est composé d'hommes en uniformes (militaires, gendarmes, douaniers) et de quelques travailleurs (instituteurs, agents de l'administration, etc.). L'organisation au sein de ce groupe est différente de celle du groupe 1. Sa création, plus récente, est composée de jeunes qui se sont retrouvés à l'école secondaire, à l'université ou dans le secteur. Ceci explique que les gens se connaissent moins bien que dans le premier groupe. Ils ont choisi de se retrouver tous les soirs chez l'un de leurs camarades vivant seul dans une cour pour boire du thé et jouer à la belote. Les thèmes de discussion portent en général sur la politique et les faits culturels.

Enfin, dans le groupe du secteur 16 (groupe 3), on retrouve pratiquement la même composition ethnique que dans le groupe 2 : mossi, dioula, bissa, samo, peul, gourounsi. Les membres y sont dans l'ensemble des étudiants, des fonctionnaires et des travailleurs. Il y a très peu d'élèves dans cette structure où l'âge des membres varie entre 20 et 35 ans. Ils se retrouvent 
tous les soirs chez l'un de leurs camarades fonctionnaires pour boire le thé et jouer aux cartes. Les membres de ce groupe se sont tous connus récemment. Les relations qui unissent ces joueurs de cartes sont donc circonstancielles, car la plupart d'entre eux ne sont pas natifs de la ville. Si certains sont là pour poursuivre leurs études secondaires ou supérieures, d'autres sont là pour des raisons professionnelles, étant donné que la majorité des services publics est concentrée à Ouagadougou.

\section{L'utilisation des langues dans les groupes de jeunes}

L'observation des pratiques langagières au sein des groupes a montré que ces comportements langagiers sont les mêmes d'un groupe à un autre. Ainsi, dans tous les groupes, on distingue deux types de situations : la situation où la pratique langagière est fortement stabilisée, et les cas où elle est variable. Dans le premier cas de figure, les locuteurs ont le choix de parler l'une ou l'autre langue sans les alterner. Dans le second cas, les jeunes ne peuvent pas maintenir les codes séparés.

\section{Les cas où le choix de la langue est stabilisé}

L'observation des pratiques langagières au sein des trois groupes montre la prépondérance de trois langues dans les interactions. Il s'agit par ordre de fréquence du français, du moore et du dioula. À ces langues interethniques, il faut ajouter l'usage sporadique de langues intra-ethniques telles que le nouni et le bisa.

Le français est utilisé au sein des groupes pour faciliter, d'une part, l'intercompréhension entre les différents membres, les groupes étant tous de composition pluriethnique, et d'autre part, pour affirmer son appartenance à la classe dominante (la classe des francophones). Langue d'aucune ethnie, le français sert également de trait d'union entre les ethnies.

La présence du moore dans les groupes est due au fait que nous sommes dans l'espace linguistique moaga, mais aussi au fait que les moorephones sont les plus nombreux dans les groupes. Quant au dioula, il doit sa place à son dynamisme. Langue véhiculaire à l'ouest du Burkina Faso, il est de plus en plus parlé à Ouagadougou à cause du phénomène migratoire. Cette migration est entretenue par les élèves et les commerçants dioula qui font des va-et-vient entre Ouagadougou et Bobo-Dioulasso.

Pour mesurer la fréquence d'utilisation des langues, nous avons tenu compte de la longueur des interventions des enquêtés dans l'une ou l'autre langue au cours des interactions.

À titre illustratif, voici une conversation enregistrée dans le groupe 1 et qui montre la prépondérance du français dans une interaction entre jeunes. 
La conversation portait sur la manière dont un homme doit se comporter devant une femme :

L1 : «Tu vois, la femme c'est comme un enfant. Faut la flatter, mais pas passer son temps à la bastonner hein. »

L2 : «Moi, je l'ai trop flattée, y a même pas. Non, non c'est facile même quoi. Parce que la manière dont toi-même tu as parlé là, tu as utilisé les sous.»

L3 : «On utilise pas les sous pour avoir une femme. Il y a des éléments, plus les sous. Parce que des fois, elle vient que oué, ta copine là dit que ça. Je dis que non, Aïcha, faut pas prendre tes copines... On n'est pas pareil sur la terre là. Si c'était ça, si on était pareil là, est-ce que Kanazoé allait passer, on n'allait jamais indiquer Kanazoé. »

L1 : «Le problème c'est toi-même tu arrives à vaincre la go là tu vois ? C'est-à-dire c'est dans la tête qu'il faut que je... non, non, c'est toi-même qui dois changer. Parce que tu as mis l'argent en avant. Or généralement dès que tu fais passer l'argent en avant, mais elle dit que lui là, il est bête... oué oué, voilà. Des conneries comme ça quoi. Tu mets l'argent en avant. »

L2 : "Mais non si elle même, elle me demande quelque chose, je la donne. Même si c'est pas sur place, deux jours après. Mais l'argent là, c'est pas avec elle que moi... Je gaspille hein ! Moi je gaspille l'argent avec mes potes. »

L3 : «Est-ce que c'est vrai ?»

B2 : «Mais le problème, écoute... l'appui là, ça a démarré y a même pas longtemps. Avec la P50 là. Parce que, non, un jour, elle m'a dit que, elle, elle veut sortir avec quelqu'un. D'abord... je l'ai... tu peux partir quoi. Et puis, mois je suis parti, moi je suis allé au restaurant avec mes potes quoi... Salia m'a dit que tu es en train de commettre une erreur. »

On note au cours de cet échange que toute la discussion s'est déroulée en français, ce qui est un témoignage du dynamisme de cette langue. À aucun moment, les intervenants n'ont eu recours à une langue nationale. Dans les cas où les jeunes utilisent les langues nationales, ils le font dans un but essentiellement ludique :

L2 : «Le jour là, la go m'a menti que c'est l'ami de son grand frère. Et le grand frère me dit que lui, il ne connaît même pas le gars. »

L1 : «keme fila di san ("deux mille francs/donner") mon frère. »

L2 : «Attention, il y a la tension actuellement donc moi, je ne veux pas rire. »

L1 : « Il faut te calmer... tu vas voir d'ici là, la situation va s'arranger. » 
L2 : "Ah tu vois le dogo ("petit frère") là je ne sais même plus ce qu'il faut faire. »

L3 : «Il suffit tout simplement que tu freines un peu... et tu prends tes distances, ce que tu avais l'habitude de donner tu arrêtes un peu. $\mathrm{Tu}$ vas voir que quand elle va faire les comparaisons entre ce qu'elle recevait de l'autre et ce que toi tu lui donnais maintenant, elle va faire son choix... y aura la réalité. »

L2 : "Ousséni ji dija m ba mi de ni ma di m bi do ki blese sisan ("eau/ donne/moi/boire/sinon/je vais le blesser tout de suite") tu entends ?»

L1 : «Toi Henri Zongo même tu es violent. »

Dans cette interaction, les interventions en français sont plus longues que celles en langue nationale dioula. Cette situation nous conduit à dire que les jeunes préfèrent davantage utiliser le français que les langues nationales.

Il importe également de signaler que nous n'avons pas observé dans les groupes des cas où des personnes se sont exprimées entièrement dans une langue nationale.

\section{Les cas où le choix de la langue n'est pas stabilisé}

Les pratiques langagières au sein des trois groupes permettent de se rendre compte qu'il y a des situations où les locuteurs utilisent de manière alternée le français et les langues nationales.

Les alternances de codes au sein des groupes se présentent de deux manières : soit les jeunes commencent leur discours en langues nationales et le terminent en français, soit ils le commencent en français et le terminent en langues nationales (moore, dioula).

Pour le premier cas, voici deux exemples:

dioula/français

L1 : «a bedi (“qu'est-ce qu'il y a") y a le bambou ici ? y a quelle nouvelle?»

L2 : « $i$ tu be mi (“où tu étais ?”) et puis c'est maintenant que où étais tu tu arrives?»

moore/français

L1 : «a bebeto poglame ("Bebeto est bléssé”) c'est pourquoi Troussier ne veut pas le classer.»

L2 : «m pa teed ti yaa sid ye ("je ne crois pas que ce soit la vérité"). Troussier ne veut pas le classer tout simplement parce qu'il n'a pas les bras longs. » 
Pour les discours commençant en français, nous avons les exemples suivants :

français/dioula

L3 : «Lundi c'est un jour hein jo lo y a fo ko a ma di ("qui a dit que c'est pas bon"). »

L4 : «C'est moi parce que je suis élève mais ele ti ta lekol la ("toi tu ne vas pas à l'école").»

français/moore

L3 : «Bonsoir les amis, yaa boe kibar $n$ be ("Quelles nouvelles y at-il ?"). »

L4: «Ben ça va fomeng ye ti bum ka be ye ("toi même, tu vois qu'il n'y a rien"). »

Un autre élément sur les usages linguistiques, qu'il faut signaler, est l'utilisation des expressions argotiques dans les groupes. À ce sujet, nous avons relevé quelques expressions :

go pour fille

mec pour garçon

fall pour cigarette

maga pour frapper

badou pour manger

pia pour argent

coco pour escroc

guaper pour droguer

wack pour gris-gris

Ces formes présentées ci-dessus sont utilisées à des fins ludiques.

\section{Les facteurs qui déterminent le choix des langues au sein des groupes}

D'après les six jeunes interrogés (deux par groupe), le choix des langues dans les groupes est lié à la pression sociale et à certaines habitudes acquises.

Le déterminisme (Gardner-Chloros 1985 : 34, citant Ludi) social, c'està-dire la situation où le choix de langue est déterminé entièrement par la société, s'observe dans le cas où le choix de la langue est durable.

Il ressort de nos entretiens que trois raisons expliqueraient l'utilisation de la langue française au sein des groupes : le besoin d'identification à un groupe, la recherche d'un emploi et l'effet de mode.

L'utilisation du français est liée au désir d'appartenir à la communauté francophone. En effet, dans un pays où la langue officielle est le français, 
les affaires politiques et économiques sont réglées en français. Ainsi, ceux qui ne parlent pas cette langue sont exclus des cercles des décisions importantes concernant la vie de la nation. L'amélioration de la pratique langagière en français aide aussi les jeunes à être plus compétitifs lorsqu'ils passent des concours ou des tests lors des recrutements de travailleurs. Selon nos enquêtés, pour prétendre à un emploi de gardien à la fonction publique aujourd'hui, il faut parler français. Et plus encore, mieux on parle français, plus on a la chance d'avoir un emploi bien rémunéré. Donc, parler français confère aux jeunes une valorisation sociale.

Il faut ajouter également que l'effet de mode joue un rôle déterminant dans la décision des jeunes d'utiliser le français. Selon eux, celui qui ne parle pas français ne peut pas évoluer dans la société actuelle. Pratiquer le français est synonyme de modernité, d'appartenance à la classe dirigeante $\mathrm{du}$ pays. Ceux qui ne parlent que les langues nationales sont traités d' « archaïques », de « villageois », d' « analphabètes ».

Mais cette utilisation du français au sein des groupes est parfois une contrainte, car il n'y a pas de langue nationale pratiquée par tout le monde.

On voit donc que parler français est un «signum social». Nul ne veut être traité d'analphabète : ce qui est, pour les gens, synonyme d'ignorant, d'inculte, de paysan, etc.

L'usage des langues moore et jula dans les groupes n'est pas dû au nombre de leurs locuteurs mais plutôt à leur caractère véhiculaire. En effet, ces langues sont utilisées dans l'administration, la santé, etc., à côté du français. De plus, elles procurent un certain nombre d'avantages, surtout quand on se rend au marché pour faire ses achats. Dans ce lieu, où la plupart des vendeurs parlent moore et dioula, parler l'une des langues c'est établir une connivence avec eux. Ce qui en même temps permet d'obtenir des produits à des prix abordables. La situation est tout autre pour les clients qui choisissent de s'exprimer en français. Dans ce cas, les vendeurs n'hésitent pas à majorer les prix, car pour eux tous ceux qui parlent français sont des étrangers ou des personnes nanties ou des hauts cadres de l'administration (compte tenu du statut du français).

On note donc que ce n'est pas le souci d'apprendre la culture de l'autre qui guide le choix des langues véhiculaires au sein des groupes, mais plutôt le désir d'obtenir des avantages matériels qui amène les uns et les autres à être bilingues.

En ce qui concerne l'utilisation des alternances de codes dans les groupes, elle fait désormais partie intégrante des habitudes langagières de nos enquêtés. D'après nos informations, le passage d'une langue à une autre est souvent inconscient.

Cependant, dans certains cas, l'alternance des codes a des fonctions ludiques : pour nos enquêtés, il y a certaines expressions qui peuvent davantage amuser les gens quand elles sont dites en moore ou jula. Dans d'autres cas, elle peut avoir des fonctions phatiques (attirer l'attention de l'interlocuteur dans un contexte donné) : dans un groupe de personnes parlant français, 
l'alternance de code langue nationale/français peut avoir pour but de faire un clin d'œil au locuteur de son groupe ethnique. On pourrait comparer l'alternance ici à la sonnerie de téléphone qui signale le désir de quelqu'un de s'entretenir avec une tierce personne.

Tout ceci concerne les alternances langues nationales/français. L'alternance français/langues nationales, est souvent, quant à elle, utilisée pour combler un vide sémio-culturel. Selon nos enquêtés, elle permet de dire ce que l'on n'aurait pu dire en moore ou en jula sans susciter une réprobation de la part de ses interlocuteurs. Par exemple, pour parler de l'amour et de la mort, les jeunes préféreront emprunter des termes au français, car ils ne sont pas «tabous » dans la culture française.

\section{Les représentations des langues}

La répartition des fonctions assignées aux langues au Burkina Faso ne permet pas à tous les Burkinabè de participer de manière active à l'effort de construction nationale. Dans le pays, où français et langues nationales coexistent, le français étant la langue officielle, il est omniprésent dans la vie politique, économique et culturelle de la nation. Il assume donc des fonctions très importantes :

- dans le fonctionnement des institutions étatiques. Ce moyen de communication permet aux Burkinabè de régler leurs problèmes juridiques, médicaux, administratifs, etc. ;

- dans le travail, car pour prétendre à un emploi rémunéré, il faut posséder un minimum de connaissance dans cette langue;

- dans l'enseignement. En effet, cette langue est le véhicule de l'enseignement pour tous les enfants scolarisés;

- dans l'intercommunication. Il sert de trait d'union ente les différents groupes ethniques du pays. De plus, il permet aux Burkinabè d'établir des contacts avec le monde extérieur et principalement avec les habitants des autres pays francophones.

Quant aux langues nationales, elles ne jouent que deux fonctions : la fonction de communication intra-ethnique et celle de communication interethnique entre les différentes communautés linguistiques.

$\mathrm{Au}$ regard de cette répartition fonctionnelle des langues, on note qu'il existe une diglossie entre le français et les langues nationales. Le français confère à ses locuteurs le pouvoir de direction des affaires de l'État, et les langues nationales ne donnent à leurs locuteurs que des privilèges mineurs.

L'observation des comportements langagiers dans les groupes montre que la dynamique des langues, telle que nous venons de la décrire, a une influence sur les pratiques langagières des jeunes. 
Compte tenu du caractère composite de la ville, le français sert de lingua franca entre les représentants des différents groupes ethniques à la recherche d'un emploi. C'est ce que résument les propos suivants des enquêtés :

EN1 : «Vous savez, le français est une langue plus bénéfique. Si tu vas hors de la ville de Ouagadougou, tu peux communiquer avec les gens en français. Ce que le moore par exemple ne permet pas de faire. Et même en ville ici, le français nous permet de communiquer sans problème. »

EN2 : «Vous voyez que dans le groupe, les gens appartiennent à des groupes ethniques divers. S'il n'y avait pas le français, ça allait être difficile de se comprendre. Je dis donc que le français nous permet de communiquer plus facilement. »

EN3 : «Aujourd'hui si vous n'avez pas fait les bancs, oui excusez-moi le terme, vous avez peu de chance d'obtenir un travail. Pour avoir un simple petit emploi salarié, on va demander de parler français. »

EN4 : «Vous savez aujourd'hui quelqu'un qui n'a pas été à l'école ne peut pas avoir un emploi à la fonction publique. C'est pourquoi tout le monde cherche à aller à l'école pour parler bien le français pour avoir la chance d'avoir un emploi un jour. Sans le français on ne peut rien faire.»

On note donc que le mythe du français-outil de travail subsiste encore à Ouagadougou dans les groupes de jeunes, quand bien même un nombre croissant de diplômés sont actuellement sans emploi dans le pays. Cela est dû au fait que, jusqu'à présent, c'est la langue opérante sur le marché du travail au Burkina Faso. À ce propos, «entre deux personnes qui cherchent un emploi manuel dans l'administration par exemple, l'on prendra celui qui peut s'exprimer en français même médiocrement pour faciliter la communication entre les demandeurs de services et lui » (Napon 1998: 85).

À travers les jugements de nos enquêtés, le français apparaît comme un outil de promotion sociale, car il permet, d'une part, aux francophones d'obtenir un emploi, et, d'autre part, de participer de manière active au développement du pays :

EN5 : «Vous voyez que le pays est dirigé par ceux qui parlent français. Les autres ne font que suivre ce qu'ils disent sans broncher, car ils ne comprennent pas les discours qui sont faits en français.»

EN6 : « Pour moi, j'ai constaté que si tu ne parles pas français, les gens ne te considèrent même pas. On dirait que ceux qui ne parlent pas français n'ont pas un point de vue à donner sur la vie du pays. »

Même si les jeunes affirment que l'avenir des langues nationales est incertain dans le pays, ils restent néanmoins attachés aux langues locales 
pour un certain nombre de raisons dont l'intégration au groupe ethnique et la recherche d'une identité.

EN1 : «Vous savez, les langues nationales sont importantes pour nous. Si tu parles ta langue maternelle, tu ne perds pas tes racines. Tout le monde te respecte également parce qu'on sait que tu connais ton origine ethnique. »

EN3 : «Vous savez, les langues nationales sont importantes, car c'est à travers elles que nous découvrons notre identité. Elles permettent aussi aux gens de s'intégrer facilement dans leur groupe ethnique mais aussi de rester en contact avec les parents restés au village. »

Compte tenu des réalités du pays, les jeunes souhaitent que les langues nationales soient introduites également à l'école, ne serait-ce qu'à titre de matière pour valoriser les patrimoines culturel et national. Mais ils sont tous unanimes pour affirmer qu'il est difficile d'utiliser les langues nationales comme des outils d'enseignement à cause de leur nombre très élevé (soixante langues nationales). Faut-il prendre toutes les langues ou choisir certaines d'entre elles ? Face à cette situation, ils préfèrent qu' on maintienne le français comme langue d'enseignement pour éviter de créer des conflits linguistiques entre les locuteurs des langues locales.

Cette inquiétude des jeunes n'est pas fondée dans la mesure où actuellement dans le pays un certain nombre d'expériences concluantes sont menées en matière d'alphabétisation et d'enseignement bilingue langues nationales/ français (écoles satellites, centres d'éducation de base non formelle, programme d'éducation bilingue accélérée de l'association Manegdbzanga, etc.). En réalité, les jeunes se montrent méfiants vis-à-vis de l'introduction des langues nationales dans le système éducatif, car aucun avantage socioéconomique n'est associé à l'apprentissage de ces langues. Ils ne trouvent donc aucun intérêt à apprendre ces langues à l'école étant donné qu'ils les pratiquent déjà en famille.

L'observation des comportements langagiers dans les groupes de jeunes à Ouagadougou a montré que trois types de langues sont utilisées avec une inégale importance dans les interactions entre jeunes :

- le français (langue officielle),

- les langues interethniques (moore, dioula),

- les langues intra-ethniques (bissa, nouni).

Elle a permis également de se rendre compte que dans tous les groupes, les jeunes sont au moins bilingues: ils parlent tous au moins une langue 
nationale en plus du français. Les trilingues se rencontrent au sein des locuteurs des langues intra-ethniques qui eux, parlent la langue maternelle, une langue interethnique (moore ou dioula) et la langue française.

En ce qui concerne le choix des langues, il est tributaire du rapport de force qui existe entre le français et les langues nationales et des thèmes de discussions. Ainsi, dans les cas où le choix de la langue est durable, les jeunes ont recours de manière systématique au français. Mais dans les situations où il $\mathrm{y}$ a des alternances de codes, le choix de commencer le discours dans telle ou telle langue est lié au thème de la discussion et au désir de détendre l'atmosphère au sein du groupe.

L'examen des représentations des langues montre, quant à lui, que les jeunes, en ville, ont jeté tout leur dévolu sur le français au détriment des langues nationales. Cette langue étant la langue de la communication interethnique, du travail et de la promotion sociale.

Mais, malgré cet attachement au français, les jeunes ne balaient pas du revers de la main les langues nationales qui ne confèrent que peu de privilèges à leurs locuteurs. Au contraire, ils souhaitent qu'une attention particulière soit accordée par les responsables étatiques aux langues nationales, car elles sont les langues de l'identité et de l'intégration au groupe ethnique.

En somme, ils souhaitent que les rapports entre les langues nationales et le français soient de type complémentaire au Burkina Faso. Ce qui passe par la pratique du français pour une grande partie de la population burkinabè et l'attribution d'un nouveau statut aux langues nationales. Ainsi, le français pourrait continuer à être la langue de l'enseignement du pays, et les langues nationales les véhicules de la culture nationale.

Université de Ouagadougou, Ouagadougou.

\section{BIBLIOGR APHIE}

Caitucoli, C., dir.

1993 Le français au Burkina Faso, Rouen, Université de Rouen.

Chaudenson, R.

1990 Des langues et des villes, Paris, Didier Érudition.

Chaudenson, R. (dir.)

1991 La francophonie: représentations, réalités, perspectives, Paris, Didier Érudition. 
Coulibaly, P.

1979 «Enquêtes sur les jeunes acculturés », in G. MANESSY \& P. WALD, Plurilinguisme, normes, situations, stratégies, Paris, L'Harmattan : 189-199.

Demaziere, D. \& Dubar, C.

1997 Analyser les entretiens biographiques. L'exemple de récits d'insertion, Paris, Nathan.

Dumont, P.

1983 Le français et les langues africaines au Sénégal, Paris, L’Harmattan.

Eloy, M. (dir.)

1995 La qualité de la langue. Le cas du français, Paris, Honoré Champion.

GARDNER-Chloros, P.

1985 Choix et alternance des langues à Strasbourg, Thèse de $3^{\mathrm{e}}$ cycle, Strasbourg, Université Louis Pasteur-Strasbourg I.

Gerbault, J.

1990 «Modes d'appropriation langagière en république centrafricaine », Bulletin du Centre d'études sur le plurilinguisme, 11 : 35-70.

Institut d'études et de recherches interethniques et interculturelles (Ideric)

1994 « À propos du français en Afrique. Questions de normes », Bulletin du Centre d'études sur le plurilinguisme, numéro spécial.

PRIGNITZ, G.

1983 « Si tu connais pas gros français-là, tu gagnes pas gros », ronéo.

NAPON, A.

1992 Étude du français des non-lettrés au Burkina Faso, Doctorat nouveau régime, Rouen, Université de Rouen.

1995 «Les inscriptions sur les enseignes à Ouagadougou : un exemple de gestion de la langue par ses locuteurs ", Annales de l'Université de Onagadougou, série A, vol. VII : 1-18.

1998 «Les représentations de la langue française à Ouagadougou », IAPETUS, 1 : 81-91.

RÉSUMÉ

Nous nous intéressons ici à la question de la gestion des langues dans les groupes de jeunes en milieu urbain et principalement dans la ville de Ouagadougou. II tente de montrer comment les jeunes, tiraillés entre la tradition (véhiculée par les langues nationales) et la modernité (véhiculée par le français), s'organisent pour satisfaire leurs différents besoins de communication. Ainsi, il apparaît que les comportements 
langagiers des jeunes sont les mêmes d'un groupe à un autre : utilisation prépondérante du français dans les interactions et recours à l'alternance des codes français/ langues nationales quand ils ont envie de s'amuser ou d'attirer l'attention d'un interlocuteur dans un contexte donné.

\section{ABSTRACT}

Language Behaviours of Youth Group in Urban Area. The Case of Ouagaduougou. - The paper addresses the issue of language management among urban youth groups, especially in Ouagadougou. It attempts to show how, torn between tradition (conveyed by national languages) and modernity (conveyed by French), young people cope with their different communication needs. Thus, it would seem that the language behavior of young people is similar from one group to another: preeminent use of French and recourse to French/English code alternation when they want to play or to draw the attention of the person they are speaking to in a given context.

Mots-clés/keywords : comportements langagiers, groupes de jeunes, milieu urbain, modernité, tradition/language behavior, youth groups, urban area, modernity. tradition. 